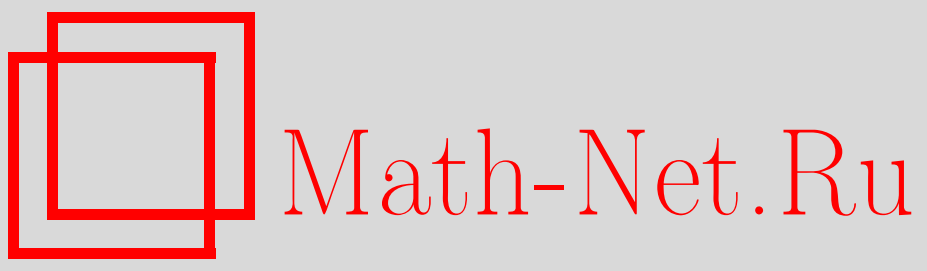

А. А. Ахметшин, Ю. С. Вольвовский, Динамика нулей конечнозонных решений уравнения Шрёдингера, Функи. анализ и его прил., 2001, том 35, выпуск 4, 8-19

DOI: https://doi.org/10.4213/faa268

Использование Общероссийского математического портала MathNet.Ru подразумевает, что вы прочитали и согласны с пользовательским соглашением

http://www . mathnet.ru/rus/agreement

Параметры загрузки:

IP : 54.92 .164 .108

26 апреля 2023 г., 10:24:56

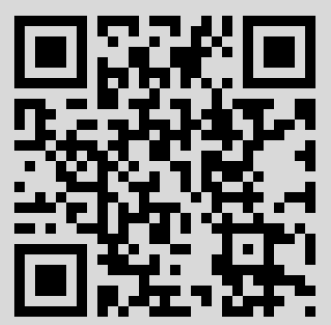




\title{
Динамика нулей конечнозонных решений уравнения Шрёдингера*
}

\author{
(c) 2001. А. А. Ахметшин, Ю. С. ВольВовСКий
}

\section{§1. Введение}

Недавно ван Дижен и Пушманн заметили, что динамика нулей $n$-солитонного решения уравнения Шрёдингера с безотражательным потенциалом удовлетворяет рациональной системе Рейсенаарса-Шнайдера с добавленным гармоническим членом ([1]; о системе РШ см. [2]). Упомянутая динамика была давно описана, хотя и в несколько ином виде; поэтому данный результат выглядит неожиданным.

В [3] было показано, что решение уравнения Шрёдингера

$$
\left(\partial_{x}^{2}-u(x)\right) \psi(x, Q)=E \psi(x, Q)
$$

с конечнозонным потенциалом $u(x)$ является корректно определенной функцией на гиперэллиптической кривой

$$
y^{2}=R_{g}(E)=\prod_{i=1}^{2 g+1}\left(E-E_{i}\right), \quad Q=(y, E) .
$$

Проекции $\zeta_{j}=\zeta_{j}(x)$ нулей этой функции на плоскость переменной $E$ удовлетворяют уравнениям Дубровина [4]:

$$
\frac{\partial \zeta_{s}}{\partial x}=\frac{2 \sqrt{R_{g}\left(\zeta_{s}\right)}}{\prod_{j \neq s}\left(\zeta_{s}-\zeta_{j}\right)} .
$$

Заметим, что эти уравнения содержат параметры кривой. Аналоги уравнений Дубровина выполняются и для частично вырожденных гиперэллиптических кривых (последние описываются тем же уравнением, где некоторые $E_{i}$ совпадают), и, в частности, для полностью вырожденных кривых (которые представляют собой сферу Римана с $n$ парами отождествленных точек). В последнем случае, как было показано в [1], параметры кривой могут быть исключены из системы. Получающаяся таким образом система оказывается системой дифференциальных уравнений, записанных исключительно в терминах нулей соответствующей функции. Она совпадает с системой Рейсенаарса-Шнайдера и как следствие является гамильтоновой. Выражения для параметров кривой служат ее первыми интегралами.

В настоящей работе мы применяем эти идеи к случаю потенциалов, отвечающих кривым с произвольной степенью вырождения. Мы рассматриваем решения $\psi(x, Q)$ соответствующих уравнений Шрёдингера, используя при этом алгеброгеометрический подход, предложенный Кричевером (см. [5]). Говоря о динамике нулей решений $\psi(x, Q)$ на данных кривых, мы подразумеваем их зависимость

*Работа выполнена при поддержке CRDF, грант RP1-2102. 
от переменной $x$. Мы показываем, что поведение нулей функции $\psi(x, Q)$ описывается динамической системой, которая является интегрируемой гамильтоновой системой $n$ частиц на гиперэллиптической кривой с выколотой точкой. Переменными типа «угол» для нее служат аналоги компонент отображения Абеля.

Во втором параграфе мы изучаем простейший случай, когда гиперэллиптическая кривая является эллиптической кривой с самопересечениями. В третьем параграфе мы обобщаем полученные результаты на случай произвольной кривой. Наиболее громоздкие доказательства вынесены в приложения I и II.

Авторы глубоко признательны профессору И. М. Кричеверу за постоянное внимание к этой работе.

\section{§2. Эллиптический случай}

Мы начнем с изложения необходимых фактов из теории конечнозонного интегрирования. Рассмотрим эллиптическую кривую Г, заданную уравнением

$$
y^{2}=E^{3}-g_{2} E-g_{3} .
$$

Она компактифицируется на бесконечности одной точкой, которую мы будем обозначать $\infty$. Единственный с точностью до умножения на константу голоморфный дифференциал на $\Gamma$ имеет вид $\omega^{h}=(d E) / y$. Пусть на $\Gamma$ выбран базис циклов $a \circ b=1$, и пусть $2 \omega_{1}$ и $2 \omega_{2}$ являются соответственно $a$ - и $b$-периодами дифференциала $\omega^{h}$. Тогда отображение Абеля, заданное формулой

$$
A: P \mapsto z=\int_{\infty}^{P} \omega^{h}
$$

позволяет идентифицировать кривую $\Gamma$ с тором $\widehat{\Gamma}=\mathbb{C} / \mathbb{Z}\left[2 \omega_{1}, 2 \omega_{2}\right]$.

Решетке $\mathbb{Z}\left[2 \omega_{1}, 2 \omega_{2}\right]$ соответствуют стандартные функции Вейерштрасса (определения и свойства см. в [7])

$$
\sigma\left(z \mid \omega_{1}, \omega_{2}\right), \quad \zeta\left(z \mid \omega_{1}, \omega_{2}\right)=\frac{\sigma^{\prime}\left(z \mid \omega_{1}, \omega_{2}\right)}{\sigma\left(z \mid \omega_{1}, \omega_{2}\right)}, \quad \wp\left(z \mid \omega_{1}, \omega_{2}\right)=-\zeta^{\prime}\left(z \mid \omega_{1}, \omega_{2}\right) .
$$

Функция $\sigma(z)$ в окрестности нуля имеет разложение $\sigma(z)=z+O\left(z^{5}\right)$ и при сдвиге аргумента на период удовлетворяет соотношениям $\sigma\left(z+2 \omega_{j}\right)=e^{2 \eta_{j}\left(z+\omega_{j}\right)} \sigma(z)$, $j=1,2$, где $\eta_{j}=\zeta\left(\omega_{j}\right)$. Функция $\zeta(z)$ обладает следующей монодромией:

$$
\zeta\left(z+2 \omega_{1}\right)=\zeta(z)+2 \eta_{1}, \quad \zeta\left(z+2 \omega_{2}\right)=\zeta(z)+2 \eta_{2},
$$

а ю-функция Вейерштрасса двоякопериодична и не имеет полюсов за исключением двойного полюса в точке $z=0$.

Отображение $z \mapsto\left(E=\wp(z), y=\wp^{\prime}(z)\right)$ является обратным к отображению Абеля.

Выберем $n-1$ точек $\kappa_{1}, \ldots, \kappa_{n-1}$ на $\widehat{\Gamma}$. Следующее утверждение является типичным в теории конечнозонного интегрирования (см. [6]).

ПреДЛОЖЕНИЕ 1. Для дивизора общцего положения $D=\gamma_{1}+\cdots+\gamma_{n}$ на $\widehat{\Gamma}$ существует и единственна функция $\psi(x, z \mid D)$ со следующими свойствами:

1) $\psi(x, z \mid D)$ мероморфна на $\widehat{\Gamma}$ вне точки $z=0$ и имеет не более чем простые полюсы в точках $\gamma_{i}, i=1, \ldots, n$; 
2) в окрестности точки $z=0$ функция $\psi(x, z \mid D)$ представляется в виде

$$
\psi(x, z \mid D)=e^{x z^{-1}}\left(1+\sum_{s=1}^{\infty} \xi_{s}(x) z^{s}\right) ;
$$

3) $\psi\left(x, \kappa_{i} \mid D\right)=\psi\left(x,-\kappa_{i} \mid D\right)$.

ДокАЗАТЕЛЬСтво. Единственность функции с перечисленными свойствами следует непосредственно из теоремы Римана-Роха. Для доказательства существования положим

$$
\psi(x, z \mid D)=e^{\zeta(z) x} \frac{\prod_{i=1}^{n} \sigma\left(z-z_{i}(x)\right)}{\prod_{s=1}^{n} \sigma\left(z-\gamma_{s}\right) \prod_{i=1}^{n} \sigma\left(z_{i}(x)\right)},
$$

где $z_{i}(x)-$ некоторые функции от $x$. Определенная таким образом функция будет двоякопериодичной, если и только если выполняется равенство $\sum_{i=1}^{n} z_{i}(x)=$ $x+\sum_{s=1}^{n} \gamma_{s}$. Данное условие и равенства $\psi\left(x, \kappa_{i} \mid D\right)=\psi\left(x,-\kappa_{i} \mid D\right)$ образуют систему $n$ уравнений на функции $z_{i}(x)$. Для дивизора $D$ общего положения эта система невырожденна. Решение в этом случае единственно с точностью до перестановок и однозначно определяет функцию $\psi(x, z \mid D)$.

ЗАмечАНИЕ. Функцию $\psi(x, z \mid D)$ принято называть функцией Бейкера-Ахиезера. В общем случае она определена на самой кривой $Г$. В эллиптическом случае удобно использовать изоморфизм между $\Gamma$ и ее якобианом $\widehat{\Gamma}$, что позволяет написать явное выражение для $\psi(x, z \mid D)$ с помощью функций Вейерштрасса.

СледствиЕ 1. Функция Бейкера-Ахиезера $\psi(x, z \mid D)$ удовлетворяет уравнению Шрёдингера

$$
\left(\partial_{x}^{2}+u(x)\right) \psi(x, z)=\wp(z) \psi(x, z)
$$

с потенциалом $u(x)=-2 \sum_{i=1}^{n} \wp\left(z_{i}(x)\right) z_{i}^{\prime}(x)$.

ДокАЗАТЕЛЬСтво. Рассмотрим функцию $\psi_{0}(x, z \mid D)=\left(\partial_{x}^{2}+u(x)-\wp(z)\right) \psi(x, z \mid D)$. Легко проверить явно, что функция $\psi(x, z \mid D)+\psi_{0}(x, z \mid D)$ обладает всеми свойствами, определяющими $\psi(x, z \mid D)$. Единственность функции Бейкера-Ахиезера влечет за собой равенство $\psi_{0}(x, z \mid D)=0$.

Теорема 1. Нули $z_{i}=z_{i}(x)$ функизии $\psi(x, z \mid D)$ подинняются системе дифференциальных уравнений

$$
z_{i}^{\prime \prime}=\sum_{k \neq i} z_{i}^{\prime} z_{k}^{\prime} \frac{\wp^{\prime}\left(z_{i}\right)+\wp^{\prime}\left(z_{k}\right)}{\wp\left(z_{i}\right)-\wp\left(z_{k}\right)}, \quad i=1, \ldots, n .
$$

ДокАЗАТЕЛЬСтво. Уравнения (4) возникают, если поделить (3) на $\psi(x, z \mid D)$ и сравнить вычеты левой и правой частей полученного равенства в точках $z=z_{i}$.

ЗАмЕчАниЕ. Теорема 1 предоставляет широкий класс решений системы (4), приходящих из алгебро-геометрических данных. Соображения размерности показывают, что таким образом получаются все решения. Другими словами, мы могли бы начать с произвольного решения системы (4) и показать, что соответствующая ему эллиптическая функция (2) удовлетворяет уравнению Шрёдингера.

Начиная с этого момента, основным объектом нашего внимания будет система (4). Эта система описывает движение $n$ частиц на кривой $\Gamma$ вне точки $\infty$ 
или, с другой точки зрения, на $\widehat{\Gamma}$ вне точки $z=0$. Введем новые переменные $\xi_{i}=\ln z_{i}^{\prime}, i=1, \ldots, n$; тогда в переменных $z_{i}, \xi_{i}$ система (4) перепишется в виде

$$
\begin{aligned}
z_{i}^{\prime} & =e^{\xi_{i}}, \\
\xi_{i}^{\prime} & =\sum_{k \neq i} e^{\xi_{k}} \frac{\wp^{\prime}\left(z_{i}\right)+\wp^{\prime}\left(z_{k}\right)}{\wp\left(z_{i}\right)-\wp\left(z_{k}\right)}, \quad i=1, \ldots, n .
\end{aligned}
$$

ПреДЛоЖение 2. Динамическая система (5) является гамильтоновой по отношению к гамильтониану $H=\sum_{i=1}^{n} e^{\xi_{j}}$ и 2-форме

$$
\omega=\sum_{i=1}^{n} d z_{i} \wedge d \xi_{i}-\frac{1}{2} \sum_{i \neq j} \frac{\wp^{\prime}\left(z_{i}\right)+\wp^{\prime}\left(z_{j}\right)}{\wp\left(z_{i}\right)-\wp\left(z_{j}\right)} d z_{i} \wedge d z_{j} .
$$

Доказательство заключается в непосредственной выкладке, которую мы опускаем.

Заметим, что

$$
\begin{aligned}
\omega & =\sum_{i=1}^{n} d z_{i} \wedge d \xi_{i}-\sum_{j \neq i} \frac{\wp^{\prime}\left(z_{i}\right)}{\wp\left(z_{i}\right)-\wp\left(z_{j}\right)} d z_{i} \wedge d z_{j} \\
& =\sum_{i=1}^{n} d z_{i} \wedge d \xi_{i}+\sum_{j \neq i} d z_{i} \wedge \frac{\wp^{\prime}\left(z_{j}\right) d z_{j}-\wp^{\prime}\left(z_{i}\right) d z_{i}}{\wp\left(z_{j}\right)-\wp\left(z_{i}\right)} \\
& =\sum_{i=1}^{n} d z_{i} \wedge d \xi_{i}+\sum_{i \neq j} d z_{i} \wedge d\left(\ln \left(\wp\left(z_{j}\right)-\wp\left(z_{i}\right)\right)\right)=\sum_{i=1}^{n} d z_{i} \wedge d \rho_{i},
\end{aligned}
$$

где

$$
\rho_{i}=\xi_{i}+\sum_{j \neq i} \ln \left(\wp\left(z_{j}\right)-\wp\left(z_{i}\right)\right) .
$$

Вид первых интегралов системы (4) подсказывает алгебро-геометрическая конструкция. Из условий $\psi\left(x, \kappa_{s} \mid D\right)=\psi\left(x,-\kappa_{s} \mid D\right)$ (свойство 3 предложения 1) вытекают равенства

$$
\sum_{j=1}^{n} \frac{z_{j}^{\prime}}{\wp\left(\kappa_{s}\right)-\wp\left(z_{j}\right)}=0, \quad s=1, \ldots, n-1 .
$$

Будучи переписаны в виде

$$
\sum_{i=1}^{n} z_{i}^{\prime} \prod_{j \neq i}\left(\wp\left(z_{j}\right)-\wp\left(\kappa_{s}\right)\right)=0, \quad s=1, \ldots, n-1,
$$

эти равенства означают, что $\widehat{\kappa}_{s}=\wp\left(\kappa_{s}\right), s=1, \ldots, n-1$, являются нулями полинома

$$
L(\lambda)=L\left(\lambda \mid z, z^{\prime}\right)=\sum_{k=0}^{n-1} H_{k}\left(z, z^{\prime}\right) \lambda^{k}=\sum_{i=1}^{n} z_{i}^{\prime} \prod_{j \neq i}\left(\wp\left(z_{j}\right)-\lambda\right) .
$$

Теорема 2. Коэффициенты $H_{k}$ полинома (7) являются интегралами движения системы (4). 
ЗАмЕчАНИЕ. Старший коэффициент $H_{n-1}\left(z, z^{\prime}\right)$ в $(7)$ с точностью до знака совпадает с гамильтонианом $H\left(z, z^{\prime}\right)$ системы (4).

Можно показать, что утверждение теоремы следует из того факта, что все решения системы (4) являются алгебро-геометрическими (коэффициенты $H_{k}$ являются симметрическими функциями нулей $\widehat{\kappa}_{s}$ полинома $\left.L(\lambda)\right)$. Мы предлагаем независимое прямое доказательство, которое приведено в приложении I.

Заметим, что $L\left(\wp\left(z_{j}\right)\right)=e^{\rho_{j}}$. Используя это равенство, можно переписать форму $\omega$ в следующем виде:

$$
\begin{aligned}
\omega & =\sum_{i=1}^{n} d z_{i} \wedge d \rho_{i}=\sum_{i=1}^{n} d z_{i} \wedge d \ln L\left(\wp\left(z_{i}\right)\right) \\
& =\sum_{i=1}^{n} \frac{1}{L\left(\wp\left(z_{i}\right)\right)} d z_{i} \wedge d\left(\sum_{s=0}^{n-1} H_{s} \wp^{s}\left(z_{i}\right)\right)=\sum_{i=1}^{n} \sum_{s=0}^{n-1} \frac{\wp^{s}\left(z_{i}\right)}{L\left(\wp\left(z_{i}\right)\right)} d z_{i} \wedge d H_{s} \\
& =\sum_{s=0}^{n-1} d\left(\sum_{i=1}^{n} \int_{E_{0}}^{\wp\left(z_{i}\right)} \frac{E^{s} d E}{L(E) y(E)}\right) \wedge d H_{s}+\sum_{s, k=0}^{n-1}\left(\sum_{i=1}^{n} \int_{E_{0}}^{\wp\left(z_{i}\right)} \frac{E^{s+k} d E}{L(E)^{2} y(E)}\right) d H_{k} \wedge d H_{s} \\
& =\sum_{s=0}^{n-1} d\left(\sum_{i=1}^{n} \int_{E_{0}}^{\wp\left(z_{i}\right)} \frac{E^{s} d E}{L(E) y(E)}\right) \wedge d H_{s},
\end{aligned}
$$

где $E_{0}-$ некоторая зафиксированная точка на комплексной плоскости переменной $E$, а функция $y(E)$ определятся уравнением (1).

Таким образом, нами доказана следующая

Tеорема 3. Переменные

$$
\varphi_{s}=\sum_{i=1}^{n} \int_{E_{0}}^{\wp\left(z_{i}\right)} \frac{E^{s} d E}{L(E) y(E)}, \quad s=0, \ldots, n-1,
$$

и коэффициенты $H_{s}$ полинома $L\left(\lambda \mid z, z^{\prime}\right)$ являются переменными типа «действие-угол» для системы (4).

Дополнительно мы хотим переписать форму $\omega$ в терминах нулей $\widehat{\kappa}_{s}, s=$ $1, \ldots, n-1$, полинома $L(\lambda)$. Для этого введем новые переменные

$$
\chi_{j}=\sum_{i=1}^{n} \int_{E_{0}}^{\wp\left(z_{i}\right)} \frac{d E}{\left(E-\widehat{\kappa}_{j}\right) y(E)}, \quad j=1, \ldots, n-1 .
$$

Определим также переменную

$$
\chi=\sum_{i=1}^{n} \int_{E_{0}}^{\wp\left(z_{i}\right)} \frac{d E}{y(E)} .
$$

Теорема 4. Определенная выше симплектическая форма $\omega$ может быть записана в следующем виде:

$$
\omega=d \chi \wedge d(\ln H)+\sum_{j=1}^{n-1} d \chi_{j} \wedge d \widehat{\kappa}_{j} .
$$


ЗАмеЧАНИЕ. Отметим особо то обстоятельство, что переменные $\left\{\chi, \chi_{j}, j=\right.$ $1, \ldots, n-1\}$ являются аналогами компонент отображения Абеля для вырожденной кривой. Таким образом, рассматриваемая нами гамильтонова структура вкладывается в общую схему, предложенную в [8] и развитую в [9].

Доказательство теоремы 4 ввиду громоздкости формул помещено в приложение II.

\section{§3. Общий случай}

Рассмотрим гиперэллиптическую кривую $\Gamma$ рода $g, g \geqslant 1$, заданную уравнением

$$
\Gamma=\left\{Q=(y, E) \mid y^{2}=R_{g}(E)=\prod_{i=1}^{2 g+1}\left(E-E_{i}\right)\right\} .
$$

Как и в эллиптическом случае, эта кривая компактифицируется в бесконечности одной точкой, которую мы обозначаем $\infty$. Кривая $Г$ двулистно накрывает комплесную плоскость переменной $E$.

Мы будем исследовать следующую систему дифференциальных уравнений на кривой $\Gamma$ :

$$
\zeta_{j}^{\prime \prime}=\frac{R_{g}^{\prime}\left(\zeta_{j}\right)}{2 R_{g}\left(\zeta_{j}\right)}\left(\zeta_{j}^{\prime}\right)^{2}+\sum_{k \neq j} \frac{\zeta_{j}^{\prime} \zeta_{k}^{\prime}}{\zeta_{j}-\zeta_{k}}\left(1+\sqrt{\frac{R_{g}\left(\zeta_{j}\right)}{R_{g}\left(\zeta_{k}\right)}}\right) .
$$

Теорема 5. Система (9) является гамильтоновой по отношению к 2-форме

$$
\omega=\sum_{j=1}^{n} \frac{d \zeta_{j}}{\sqrt{R_{g}\left(\zeta_{j}\right)}} \wedge d \rho_{j}
$$

где $\rho_{j}=\zeta_{j}^{\prime}+\sum_{k \neq j} \ln \left(\zeta_{k}-\zeta_{j}\right)$, и гамильтониану

$$
H=\sum_{i=1}^{n} \frac{\zeta_{j}^{\prime}}{2 \sqrt{R_{g}\left(\zeta_{j}\right)}}
$$

Коэффициентьь $H_{s}\left(\zeta, \zeta^{\prime}\right), s=0, \ldots, n-1$, полинома

$$
L(\lambda)=L\left(\lambda \mid \zeta, \zeta^{\prime}\right)=\sum_{k=0}^{n-1} H_{s}\left(\zeta, \zeta^{\prime}\right) \lambda^{s}=\sum_{j=1}^{n} \frac{\zeta_{j}^{\prime}}{\sqrt{R_{g}\left(\zeta_{j}\right)}} \prod_{k \neq j}\left(\zeta_{k}-\lambda\right)
$$

являются первыми интегралами системы (9). Вместе с величинами

$$
\varphi_{s}=\sum_{j=1}^{n} \int_{E_{0}}^{\zeta_{j}} \frac{E^{s} d E}{L(E) y(E)}, \quad s=0, \ldots, n-1,
$$

они образуют набор переменных типа «действие-угол» для системь (9).

ЗАмЕЧАНИЕ. Поскольку линии уровня первых интегралов не компактны, для системы (9) не существует канонического выбора переменных типа «действиеугол».

ДокАЗАТЕЛЬСтвО. Пусть функция $\wp_{g}(z)$ является решением дифференциального уравнения

$$
\wp_{g}^{\prime}=2 \sqrt{R_{g}\left(\wp_{g}\right)}
$$


Определим новые переменные $z_{j}, j=1, \ldots, n$, из условий $\zeta_{j}=\wp_{g}\left(z_{j}\right)$. В новых переменных система (9) переписывается в виде

$$
z_{i}^{\prime \prime}=\sum_{k \neq i} z_{i}^{\prime} z_{k}^{\prime} \frac{\wp_{g}^{\prime}\left(z_{i}\right)+\wp_{g}^{\prime}\left(z_{k}\right)}{\wp_{g}\left(z_{i}\right)-\wp_{g}\left(z_{k}\right)}, \quad i=1, \ldots, n .
$$

Дальнейший ход доказательства повторяет эллиптический случай (с заменой $\wp(z)$ на $\wp_{g}(z)$ и т. п.), и потому мы его опускаем.

ЗАмЕЧАНИЕ. Функция $\wp_{g}(z)$ является корректно определенным локальным параметром в конечной части кривой Г. Следовательно, система (9) описывает движение $n$ частиц на $\Gamma$ вне точки $\infty$.

Наша следующая цель - показать, что система (9) описывает динамику нулей решения уравнения Шрёдингера, построенного по кривой Г. Мы предполагаем, что число нулей $n$ превосходит род $g$ кривой Г. Такие решения строятся стандартным для теории конечнозонного интегрирования способом.

ПрЕДЛОЖЕНИЕ 3. Пусть в плоскости переменной $Е$ зафиксирован дивизор $R=\kappa_{1}+\cdots+\kappa_{n-g}$. Тогда для дивизора общего положения $D=\gamma_{1}+\cdots+\gamma_{n}$ на кривой Г существует единственная функция $\psi(x, Q \mid D, R)$, удовлетворяющая следуюшим условиям:

1) она мероморфна на кривой Г вне точки $\infty$ и имеет не более чем простые полюсы в точках $\gamma_{i}, i=1, \ldots, n$;

2) в окрестности точки $\infty$ она допускает разложение вида

$$
\psi(x, Q \mid D, R)=e^{x E^{1 / 2}}\left(1+\sum_{s=1}^{\infty} \xi_{s}(x) E^{-s / 2}\right) ;
$$

3) $\psi\left(x, \kappa_{i}^{+} \mid D, R\right)=\psi\left(x, \kappa_{i}^{-} \mid D, R\right)$, где $\kappa_{i}^{ \pm}$являются прообразами точек $\kappa_{i}$ при проекции на Е-плоскость.

ДоКАЗАТЕЛЬСтвО. Единственность такой функции (при условии, что она существует) следует непосредственно из теоремы Римана-Роха. Чтобы показать, что она существует, мы предъявим явное выражение для $\psi(x, Q \mid D, R)$ в терминах $\theta$-функций Римана.

Напомним, что $\theta$-функция Римана, отвечающая алгебраической кривой $Г$ рода $g$, - это целая функция $g$ комплексных аргументов $z=\left(z_{1}, \ldots, z_{g}\right)$, которая определяется своим разложением Фурье

$$
\theta\left(z_{1}, \ldots, z_{g}\right)=\sum_{m \in \mathbb{Z}^{g}} e^{2 \pi i(m, z)+\pi i(B m, m)},
$$

где $B=\left(B_{i j}\right), B_{i j}=\oint_{b_{i}} \omega_{j},-$ матрица $b$-периодов нормированных голоморфных дифференциалов $\omega_{j}(P)$ на $\Gamma: \oint_{a_{j}} \omega_{i}=\delta_{i j}$. Здесь $a_{i}, b_{i}-$ базис циклов на $\Gamma$ с канонической матрицей пересечений: $a_{i} \circ a_{j}=b_{i} \circ b_{j}=0, a_{i} \circ b_{j}=\delta_{i j}$.

$\theta$-функция обладает следующими свойствами монодромии по отношению к решетке $\mathscr{B}$, образованной базисными векторами $e_{i} \in \mathbb{C}^{g}$ и столбцами $B_{j}$ матрицы $b$-периодов:

$$
\theta(z+l)=\theta(z), \quad \theta(z+B l)=\exp [-i \pi(B l, l)-2 i \pi(l, z)] \theta(z),
$$


где $l$ - целочисленный вектор, $l \in \mathbb{Z}^{g}$. Многообразием Якоби или якобианом алгебраической кривой $Г$ называют комплексный тор $J(\Gamma)=\mathbb{C}^{g} / \mathscr{B}$. Отображение Абеля $\Gamma \mapsto J(\Gamma)$ переводит точку $Q$ в вектор $A(Q)$ с координатами

$$
A_{k}(Q)=\int_{Q_{0}}^{Q} \omega_{k}
$$

где $Q_{0}-$ некоторая фиксированная точка кривой $\Gamma$.

Согласно теореме Римана-Роха, для дивизоров общего положения $D=\gamma_{1}+$ $\cdots+\gamma_{n}$ и $\mathscr{R}=\kappa_{1}^{+}+\cdots+\kappa_{n-g+1}^{+}$, где $\kappa_{n-g+1}^{+}=\infty$, существуют и единственны такие мероморфные функции $r_{\alpha}(Q), \alpha=1, \ldots, n+g-1$, что $D$ является дивизором полюсов для каждой из них и выполнены условия $r_{\alpha}\left(\kappa_{\beta}^{+}\right)=\delta_{\alpha \beta}$. Имеют место следующие выражения для этих функций:

$$
r_{\alpha}(Q)=\frac{f_{\alpha}(Q)}{f_{\alpha}\left(\kappa_{\alpha}^{+}\right)}, \quad f_{\alpha}(Q)=\theta\left(A(Q)+Z_{\alpha}\right) \frac{\prod_{\beta \neq \alpha} \theta\left(A(Q)+F_{\beta}\right)}{\prod_{m=1}^{n-g+1} \theta\left(A(Q)+S_{m}\right)},
$$

где

$$
\begin{gathered}
F_{\beta}=-\mathscr{K}-A\left(\kappa_{\beta}^{+}\right)-\sum_{s=1}^{g-1} A\left(\gamma_{s}\right), \quad S_{m}=-\mathscr{K}-A\left(\gamma_{g-1+m}\right)-\sum_{s=1}^{g-1} A\left(\gamma_{s}\right), \\
Z_{\alpha}=Z_{0}-A\left(R_{\alpha}\right), \quad Z_{0}=-\mathscr{K}-\sum_{s=1}^{n} A\left(\gamma_{s}\right)+\sum_{\alpha=1}^{n-g+1} A\left(R_{\alpha}\right),
\end{gathered}
$$

а $\mathscr{K}$ является вектором римановых констант.

Пусть $d \Omega$ обозначает единственный мероморфный дифференциал на $Г$, имеющий двойной полюс в точке $\infty$, голоморфный всюду вне нее и нормированный $g$ условиями

$$
\oint_{a_{k}} d \Omega=0
$$

Рассмотрим вектор $b$-периодов $V=\left(V_{1}, \ldots, V_{g}\right)$ этого дифференциала:

$$
V_{k}=\frac{1}{2 \pi i} \oint_{b_{k}} d \Omega \text {. }
$$

Определим функции $\psi_{\alpha}(x, Q \mid D, R)$ следующими формулами:

$$
\psi_{\alpha}(x, Q \mid D, R)=r_{\alpha}(Q) \frac{\theta\left(A(Q)+x V+Z_{\alpha}\right) \theta\left(Z_{0}\right)}{\theta\left(A(Q)+Z_{\alpha}\right) \theta\left(\left(x V+Z_{0}\right)\right.} \exp \left(x \int_{R_{\alpha}}^{Q} d \Omega\right) .
$$

Нетрудно показать, что эти функции корректно определены на кривой Г. Пусть вектор $c_{\alpha}, \alpha=1, \ldots, n-g$, дает решение линейной системы

$$
\sum_{\alpha=1}^{n-g} c_{\alpha} \psi_{\alpha}\left(x, \kappa_{\beta}^{-} \mid D, R\right)+\psi_{n+g-1}\left(x, \kappa_{\beta}^{-} \mid D, R\right)=c_{\beta}, \quad \beta=1, \ldots, n-g .
$$

Тогда функция

$$
\psi(x, Q \mid D, R)=\psi_{n-g+1}(x, Q \mid D, R)+\sum_{\alpha=1}^{n-g} c_{\alpha} \psi_{\alpha}(x, Q \mid D, R)
$$

удовлетворяет требуемым трем условиям. Предложение доказано. 
СледСтвиЕ 2. Построенная выще функциия $\psi(x, Q \mid D, R)$ удовлетворяет уравнению Шрёдингера

$$
\left(\partial_{x}^{2}+u(x)\right) \psi(x, Q)=E \psi(x, Q)
$$

с потенциилом $u(x)=-2 \partial_{x} \xi_{1}(x)$.

Функция $\psi(x, Q \mid D, R)$ имеет $n$ нулей на $\Gamma$, проекции $\zeta_{1}, \ldots, \zeta_{n}$ которых на плоскость аргумента $E$ удовлетворяют уравнениям Дубровина, имеющим в данном случае следующий вид:

$$
\frac{\partial \zeta_{s}}{\partial x}=\frac{2 \sqrt{R_{g}\left(\zeta_{s}\right)} \prod_{\alpha=1}^{n-g}\left(\zeta_{s}-\kappa_{\alpha}\right)}{\prod_{j \neq s}\left(\zeta_{s}-\zeta_{j}\right)} .
$$

Можно переписать эти уравнения в матричной форме

$$
M v=e,
$$

где $v=\left(v_{1}, \ldots, v_{n}\right), e=\left(e_{1}, \ldots, e_{n}\right)$ и

$$
v_{i}=\frac{\partial_{x} \zeta_{i}}{2 \sqrt{R_{g}\left(\zeta_{i}\right)}}, \quad e_{j}=\delta_{j g},
$$

а элементы матрицы $M=\left(M_{j i}\right)$ имеют вид

$$
M_{j i}= \begin{cases}\zeta_{i}^{j-1}, & j \leqslant g, \\ 1 /\left(\zeta_{i}-\kappa_{j-g}\right), & j>g .\end{cases}
$$

Теорема 6. Проеки̧ии $\zeta_{j}(x), j=1, \ldots, n$, нулей функизии $\psi(x, Q \mid R, D)$ удовлетворяют уравнениям, которые получаются ограничением системь (9) на совместные линии уровня $g$ первых интегралов этой системь:

$$
H_{n-k}=\delta_{k g}, \quad k=1, \ldots, g \text {. }
$$

ДОКАЗАТЕЛЬСТвО. Проекции $\zeta_{j}$ нулей функции $\psi(x, Q \mid R, D)$ на $E$-плоскость удовлетворяют $n$ уравнениям, в совокупности составляющим матричное уравнение (10). Первые $g$ из них означают, что выполнены ограничения (11). Следовательно, полином $L\left(\lambda \mid \zeta, \zeta^{\prime}\right)$ имеет степень $n-g$, и оставшиеся $n-g$ уравнений системы (10) говорят, что точки $\kappa_{1}, \ldots, \kappa_{n-g}$ являются его нулями. Коэффициенты $L(\lambda)$, таким образом, не зависят от времени, т. е. $H_{j}^{\prime}(x)=0, j=1, \ldots, n$. Последний набор уравнений эквивалентен системе (9). Теорема доказана.

В заключение заметим, что для общего решения $\zeta_{j}, j=1, \ldots, n$, системы $(9)$, удовлетворяющего условиям (11), мы можем определить алгебро-геометрические данные, полагая $R$ дивизором нулей полинома $L(\lambda)$ и полагая дивизор $D$ равным

$$
\left(\sqrt{R_{g}\left(\zeta_{1}(0)\right)}, \zeta_{1}(0)\right)+\cdots+\left(\sqrt{R_{g}\left(\zeta_{n}(0)\right)}, \zeta_{n}(0)\right)
$$

Иными словами, почти все решения ограниченной системы можно получить, используя конструкцию из предложения 3. 


\section{Приложение I}

В этом приложении мы даем доказательство теоремы 2. Утверждение данной теоремы состоит в том, что коэффициенты полинома

$$
L(\lambda)=L\left(\lambda \mid z, z^{\prime}\right)=\sum_{j=1}^{n} z_{j}^{\prime} \prod_{i \neq j}\left(\wp\left(z_{i}\right)-\lambda\right)=\sum_{k=0}^{n-1} H_{k}\left(z, z^{\prime}\right) \lambda^{k}
$$

являются интегралами движения системы (4).

Явные формулы для коэффициентов $H_{k}$ таковы:

$$
H_{k}=\sum_{|J|=n-k-1}(-1)^{k} \prod_{j \in J} \wp\left(z_{j}\right)\left(\sum_{k \notin J} z_{k}^{\prime}\right),
$$

где суммирование ведется по всем подмножествам $J \subset\{1, \ldots, n\}$ мощности $n-$ $k-1$.

Мы собираемся показать, что функции $H_{k}$ не зависят от времени, т. е. $d H_{k} / d x$ $=0$. Действительно,

$$
\begin{aligned}
\frac{d(-1)^{k} H_{k}\left(z, z^{\prime}\right)}{d x}= & \sum_{J} \prod_{j \in J} \wp\left(z_{i}\right) \sum_{k \notin J} z_{k}^{\prime \prime}+\sum_{J}\left(\sum_{s \in J} \frac{\wp^{\prime}\left(z_{s}\right)}{\wp\left(z_{s}\right)} z_{s}^{\prime}\right) \prod_{j \in J} \wp\left(z_{i}\right) \sum_{k \notin J} z_{k}^{\prime \prime} \\
= & \sum_{J} \prod_{j \in J} \wp\left(z_{j}\right)\left(\sum_{k \notin J} \sum_{i \neq k} \frac{\wp^{\prime}\left(z_{k}\right)+\wp^{\prime}\left(z_{i}\right)}{\wp\left(z_{k}\right)-\wp\left(z_{i}\right)} z_{i}^{\prime} z_{k}^{\prime}\right) \\
& +\sum_{J} \prod_{j \in J} \wp\left(z_{j}\right)\left(\sum_{k \notin J} \sum_{s \in J} \frac{\wp^{\prime}\left(z_{s}\right)}{\wp\left(z_{s}\right)} z_{k}^{\prime} z_{s}^{\prime}\right) \\
= & \sum_{J} \prod_{j \in J} \wp\left(z_{j}\right)\left(\sum_{k \notin J} \sum_{s \in J}\left[\frac{\wp^{\prime}\left(z_{s}\right)}{\wp\left(z_{s}\right)}+\frac{\wp^{\prime}\left(z_{k}\right)+\wp^{\prime}\left(z_{s}\right)}{\wp\left(z_{k}\right)-\wp\left(z_{s}\right)}\right] z_{k}^{\prime} z_{s}^{\prime}\right) \\
= & \sum_{J, k \notin J, s \in J} \alpha(J, k, s),
\end{aligned}
$$

где

$$
\alpha(J, k, s)=\prod_{j \in J} \wp\left(z_{j}\right)\left[\frac{\wp^{\prime}\left(z_{s}\right)}{\wp\left(z_{s}\right)}+\frac{\wp^{\prime}\left(z_{k}\right)+\wp^{\prime}\left(z_{s}\right)}{\wp\left(z_{k}\right)-\wp\left(z_{s}\right)}\right] z_{k}^{\prime} z_{s}^{\prime} .
$$

Рассмотрим на множестве троек $\{J, k \notin J, s \in J\}$ инволюцию, отображающую $\{J, k, s\}$ в $\left\{J^{\prime}, s, k\right\}$, где $J^{\prime}=J \cup\{k\} \backslash\{s\}$. Заметим, что

$$
\begin{aligned}
\alpha(J, k, s)+\alpha\left(J^{\prime}, s, k\right)= & \prod_{j \in J \cap J^{\prime}} \wp\left(z_{j}\right) z_{k}^{\prime} z_{s}^{\prime}\left[\wp^{\prime}\left(z_{s}\right)+\wp^{\prime}\left(z_{k}\right)\right. \\
& \left.+\wp\left(z_{s}\right) \frac{\wp^{\prime}\left(z_{k}\right)+\wp^{\prime}\left(z_{s}\right)}{\wp\left(z_{k}\right)-\wp\left(z_{s}\right)}+\wp\left(z_{k}\right) \frac{\wp^{\prime}\left(z_{s}\right)+\wp^{\prime}\left(z_{k}\right)}{\wp\left(z_{s}\right)-\wp\left(z_{k}\right)}\right]=0
\end{aligned}
$$

и, следовательно, вся сумма $\sum_{J, k \notin J, s \in J} \alpha(J, k, s)$ равна нулю. Теорема доказана. 


\section{Приложение II}

Здесь представлено доказательство теоремы 4.

Рассмотрим 2-форму $\omega=\sum_{s=0}^{n-1} d \varphi_{s} \wedge d H_{s}$. Напомним, что $H_{s}=(-1)^{s} H \sigma_{n-s-1}(\widehat{\kappa})$ для $s=0, \ldots, n-1$, где $\sigma_{n-s-1}(\widehat{\kappa})$ обозначает коэффициент при $\lambda^{s}$ в полиноме $\prod_{i=1}^{n-1}\left(\lambda+\widehat{\kappa}_{i}\right)$. Через $\sigma_{n-s-2}^{j}(\widehat{\kappa})$ мы обозначим коэффициент при $\lambda^{s}$ в полиноме $\prod_{i \neq j}\left(\lambda+\widehat{\kappa}_{i}\right)$. Тогда

$$
\begin{aligned}
\omega & =\sum_{s=0}^{n-1} d \varphi_{s} \wedge d H_{s}=\sum_{s=0}^{n-2} d \varphi_{s} \wedge(-1)^{s} d\left(H \sigma_{n-s-1}(\widehat{\kappa})\right)+(-1)^{n-1} d \varphi_{n-1} \wedge d H \\
& =\sum_{s=0}^{n-1}(-1)^{s} \sigma_{n-s-1}(\widehat{\kappa}) d \varphi_{s} \wedge d H+\sum_{j=1}^{n-1} \sum_{s=0}^{n-2}(-1)^{s} H \sigma_{n-s-2}^{j}(\widehat{\kappa}) d \varphi_{s} \wedge d \widehat{\kappa}_{j} .
\end{aligned}
$$

Теперь заметим, что

$$
\begin{aligned}
\sum_{s=0}^{n-2}( & -1)^{s} H \sigma_{n-s-2}^{j}(\widehat{\kappa}) d \varphi_{s} \\
= & \sum_{s=0}^{n-2} \sum_{l=1}^{n}(-1)^{s} H \sigma_{n-s-2}^{j}(\widehat{\kappa}) d \int_{E_{0}}^{\wp\left(z_{l}\right)} \frac{E^{s} d E}{L(E) y(E)} \\
= & \sum_{l=1}^{n} d \int_{E_{0}}^{\wp\left(z_{l}\right)} \frac{\sum_{s=0}^{n-2}(-1)^{s} H \sigma_{n-s-2}^{j}(\widehat{\kappa}) E^{s}}{L(E) y(E)} d E \\
& \quad-\sum_{l=1}^{n} \int_{E_{0}}^{\wp\left(z_{l}\right)} d\left(\frac{\sum_{s=0}^{n-2}(-1)^{s} H \sigma_{n-s-2}^{j}(\widehat{\kappa}) E^{s}}{L(E) y(E)}\right) d E \\
= & \sum_{l=1}^{n} d \int_{E_{0}}^{\wp\left(z_{l}\right)} \frac{d E}{\left(E-\widehat{\kappa}_{j}\right) y(E)}+\sum_{l=1}^{n} \int_{E_{0}}^{\wp\left(z_{l}\right)} \frac{d E}{\left(E-\widehat{\kappa}_{j}\right)^{2} y(E)} d \widehat{\kappa}_{j} .
\end{aligned}
$$

Точно так же показывается, что

$$
\sum_{s=0}^{n-1}(-1)^{s} \sigma_{n-s-1}(\widehat{\kappa}) d \varphi_{s}=\sum_{l=1}^{n} d \int_{E_{0}}^{\wp\left(z_{l}\right)} \frac{d E}{H y(E)}+\sum_{l=1}^{n} \int_{E_{0}}^{\wp\left(z_{l}\right)} \frac{d E}{H^{2} y(E)} d H .
$$

Подставляя две эти формулы в (12), получаем

$$
\begin{aligned}
\omega & =\sum_{j=1}^{n-1} d\left(\sum_{l=1}^{n} \int_{E_{0}}^{\wp\left(z_{l}\right)} \frac{d E}{\left(E-\widehat{\kappa}_{j}\right) y(E)}\right) \wedge d \widehat{\kappa}_{j}+d\left(\sum_{l=1}^{n} \int_{E_{0}}^{\wp\left(z_{l}\right)} \frac{d E}{H y(E)}\right) \wedge d H \\
& =\sum_{j=1}^{n-1} d \chi_{j} \wedge d \widehat{\kappa}_{j}+d \chi \wedge d(\ln H) .
\end{aligned}
$$

Именно возможность записать форму $\omega$ в таком виде составляет утверждение теоремы 4. 


\section{ЛИТЕРАТУРА}

1. Van Diejen J. F., Puschmann H. Reflectionless Schrödinger operators, the dynamics of zeros, and the solitonic Sato formula. Duke Math. J., 104, No. 2, 269-318 (2000).

2. Ruijsenaars S. N. M., Schneider H. A new class of integrable systems and its relation to solitons. Ann. Physics, 170, No. 2, 370-405 (1986).

3. Дубровин Б. А., Матвеев В. Б., Новиков С. П. Нелинейные уравнения типа Кортевегаде Фриза, конечнозонные линейные операторы и абелевы многообразия. УМН, $\mathbf{3 1}$, вып. 1 (187), 55-136 (1976).

4. Дубровин Б. А. Периодические задачи для уравнения Кортевега-де Фриза в классе конечнозонных потенциалов. Функц. анализ и его прил., 9, вып. 3, 41-52 (1975).

5. Кричевер И. М. Потенциалы с нулевым коэффициентом отражения на фоне конечнозонных потенциалов. Функц. анализ и его прил., 9, вып. 2, 77-79 (1975).

6. Кричевер И. М. Спектральная теория «конечнозонных» нестационарных операторов Шрёдингера. Нестационарная модель Пайерлса. Функц. анализ и его прил., 20, вып. 3, 42-54 (1986).

7. Bateman H., Erdelyi A. Higher Transcendental Functions, Vol. II. McGraw-Hill, 1953.

8. Веселов А. П., Новиков С. П. О скобках Пуассона, совместимых с алгебраической геометрией и динамикой Кортевега-де Фриза на множестве конечнозонных потенциалов. ДАН СССР, 266, № 3, 533-537 (1982).

9. Krichever I. M., Phong D. Symplectic forms in the theory of solitons. In: Surv. Differ. Geom. IV, Suppl. J. Differ. Geom., 239-313 (1998).

Институт теоретической физики РАН им. Л. Д. Ландау, Columbia University

e-mail: alakhm@math.columbia.edu

Институт теоретической физики РАН им. Л. Д. Ландау,

Columbia University

e-mail: yurik@math.columbia.edu 